\title{
A 4D Hyperspherical Interpretation of $q$-space
}

\author{
A. Pasha Hosseinbor ${ }^{1}$, Moo K. Chung ${ }^{1}$, Yu-Chien $\mathrm{Wu}^{2}$, \\ Andrew L. Alexander ${ }^{1}$, and Barbara B. Bendlin ${ }^{1}$ \\ 1 University of Wisconsin-Madison, USA \\ 2 Dartmouth College, USA \\ hosseinbor@wisc.edu
}

\begin{abstract}
D $q$-space can be viewed as the surface of a $4 \mathrm{D}$ hypersphere. In this paper, we seek to develop a $4 \mathrm{D}$ hyperspherical interpretation of $q$-space by projecting it onto a hypersphere and subsequently modeling the $q$-space signal via $4 \mathrm{D}$ hyperspherical harmonics (HSH). Using this orthonormal basis, we analytically derive several quantitative indices and numerically estimate the diffusion ODF. Importantly, we derive the integral transform describing the relationship between the diffusion signal and propagator on a hypersphere. We also show that the HSH basis expends less fitting parameters than other well-established methods to achieve comparable signal and better ODF reconstructions. All in all, this work provides a new way of looking at $q$-space.
\end{abstract}

\section{Introduction}

One of the first physical applications of quantum mechanics was in solving the Schrödinger equation for the hydrogen atom. It had been solved in positionspace by Schrödinger, himself, but not in momentum-space, which is related to position-space via the Fourier transform. The momentum-space solution was of interest to quantum chemists because it could potentially reveal additional quantum mechanical insights about the hydrogen atom not found in the position space solution. Nearly a decade after Schrödinger's work, V. Fock solved the Schrödinger equation for the hydrogen atom directly in momentum-space. In his classic paper 4, Fock stereographically projected 3D momentum-space onto the surface of a 4D unit hypersphere, and after this mapping was made, he was able to show that the momentum-space hydrogen orbitals could be simply expressed in terms of 4D hyperspherical harmonics (HSH), which are the multidimensional analogues of the 3D spherical harmonics.

In diffusion MRI, analogous to momentum- and position-space in quantum mechanics, the signal decay and ensemble average propagator (EAP) are Fourier transform (FT) pairs within the $q$-space framework:

$$
P(\mathbf{k})=\int E(\mathbf{q}) e^{-2 \pi i \mathbf{q} \cdot \mathbf{k}} d^{3} \mathbf{q}
$$

where $\mathbf{k}$ is the displacement vector in EAP-space and $\mathbf{q}$ is the diffusion wavevector in signal-space. We denote $\mathbf{q}=q \mathbf{u}$ and $\mathbf{k}=k \mathbf{r}$, where $\mathbf{u}$ and $\mathbf{r}$ are $3 \mathrm{D}$ unit vectors. An interesting problem, similar to that of the hydrogen atom, is 
whether a new interpretation of $q$-space can be obtained by stereographically projecting $q$-space and EAP-space onto a hypersphere.

In this paper, following the work of Fock, we seek to develop a 4D hyperspherical interpretation of $q$-space by focusing on four things: 1) Modeling the 3D $q$-space signal in terms of the $4 \mathrm{D} \mathrm{HSH}$, which is achieved by stereographically projecting 3D $q$-space onto the surface of a $4 \mathrm{D}$ hypersphere; 2) Using this single, orthonormal basis to reconstruct the diffusion orientation distribution function $(\mathrm{ODF}) ; 3)$ Computing the familiar $q$-space metric zero-displacement probability $(P o)$ and introducing a novel hyperspherical diffusivity index; and 4) Deriving the integral transform that maps from the signal-hypersphere to the EAP-hypersphere. The last point is especially significant because the integral transform describing the relationship between any two functions individually existing on some $n$-dimensional sphere $S^{n}$, given that the two functions are FT pairs on the $(n-1)$-plane, has never been derived. Lastly, we compare the HSH basis to Bessel Fourier Orientation Reconstruction (BFOR) [5], and show that $\mathrm{HSH}$ expansion requires less fitting parameters than BFOR to achieve comparable signal and better ODF reconstructions.

\section{Methods}

\subsection{D Hyperspherical Harmonics}

Consider the 4D unit hypersphere $S^{3}$ existing in $\mathbb{R}^{4}$. The Laplace-Beltrami operator on $S^{3}$ is defined as $\Delta_{S^{3}}=\frac{1}{\sin ^{2} \beta} \frac{\partial}{\partial \beta} \sin ^{2} \beta \frac{\partial}{\partial \beta}+\frac{1}{\sin ^{2} \beta} \Delta_{S^{2}}$, where $\Delta_{S^{2}}$ is the Laplace-Beltrami operator on the unit sphere $S^{2}$. The eigenfuctions of $\Delta_{S^{3}}$ are the $4 \mathrm{D}$ HSH $Z_{n l}^{m}(\beta, \theta, \phi): \Delta_{S^{3}} Z_{n l}^{m}=-l(l+2) Z_{n l}^{m}$. The $4 \mathrm{D} \mathrm{HSH}$ are defined as [3]

$$
Z_{n l}^{m}(\beta, \theta, \phi)=2^{l+1 / 2} \sqrt{\frac{(n+1) \Gamma(n-l+1)}{\pi \Gamma(n+l+2)}} \Gamma(l+1) \sin ^{l} \beta C_{n-l}^{l+1}(\cos \beta) Y_{l}^{m}(\theta, \phi),
$$

where $(\beta, \theta, \phi)$ obey $(\beta \in[0, \pi], \theta \in[0, \pi], \phi \in[0,2 \pi]), C_{n-1}^{l+1}$ are the Gegenbauer (i.e. ultraspherical) polynomials, and $Y_{l}^{m}$ are the $3 \mathrm{D}$ spherical harmonics. The $l$ denotes the degree of the HSH, $m$ is the order, and $n=0,1,2, \ldots$, and these three integers obey the conditions $0 \leq l \leq n$ and $-l \leq m \leq l$. The number of HSH corresponding to a given value of $n$ is $(n+1)^{2}$. The HSH form an orthonormal basis on the hypersphere.

\subsection{D Stereographic Projection of $q$-space onto Hypersphere}

In order to model the $q$-space signal with the HSH, we need to map 3D $q$-space onto a $4 \mathrm{D}$ hypersphere of radius $p_{o}$, which is achieved via stereographic projection. The $q$-space coordinates are defined as $q_{x}=q \sin \theta \cos \phi, q_{y}=q \sin \theta \sin \phi$, and $q_{z}=q \cos \theta$. The coordinates of the signal-hypersphere are defined by the $4 \mathrm{D}$ 
vector $\mathbf{s}$, whose components are $s_{1}=p_{o} \sin \beta \sin \theta \cos \phi, s_{2}=p_{o} \sin \beta \sin \theta \sin \phi$, $s_{3}=p_{o} \sin \beta \cos \theta$, and $s_{4}=p_{o} \cos \beta$. The relationship between $\mathbf{q}$ and $\mathbf{s}$ is then

$$
s_{1}=\frac{2 p_{o}^{2} q_{x}}{q^{2}+p_{o}^{2}}, \quad s_{2}=\frac{2 p_{o}^{2} q_{y}}{q^{2}+p_{o}^{2}}, \quad s_{3}=\frac{2 p_{o}^{2} q_{z}}{q^{2}+p_{o}^{2}}, \quad s_{4}=\frac{p_{o}\left(q^{2}-p_{o}^{2}\right)}{q^{2}+p_{o}^{2}}
$$

According to Eq. (3), the center of $q$-space $(0,0,0)$ projects onto the south pole $\left(0,0,0,-p_{o}\right)$ of the hypersphere. As $q \rightarrow \infty$, the projection $\left(s_{1}, s_{2}, s_{3}, s_{4}\right)$ moves closer to the north pole $\left(0,0,0, p_{o}\right)$. Eq. (3) establishes a one-to-one correspondence between $q$-space and the $4 \mathrm{D}$ hypersphere.

Stereographic projection exhibits two important properties. First, it is conformal, which means it preserves angles - the angles $(\theta, \phi)$ in $q$-space are preserved in 4D hyperspherical space. However, stereographic projection does not preserve volume; in general, the volume of a region in the $3 \mathrm{D}$ plane doesn't equal the volume of its projection onto the hypersphere. In fact, the degree of volume distortion in going from a differential volume element in $q$-space $d^{3} \mathbf{q}$ to that of the hypersphere $d V=p_{o}^{3} d \Omega$ can be shown to be

$$
d^{3} \mathbf{q}=\left(\frac{q^{2}+p_{o}^{2}}{2 p_{o}}\right)^{3} \frac{1}{p_{o}^{3}} d V=\left(\frac{q^{2}+p_{o}^{2}}{2 p_{o}}\right)^{3} d \Omega=\left(\frac{p_{o}}{1-\cos \beta}\right)^{3} d \Omega,
$$

where $d \Omega=\sin ^{2} \beta \sin \theta d \beta d \theta d \phi$.

\subsection{Diffusion Signal Modeling via HSH Basis}

Stereographically projecting $q$-space onto the hypersphere results in the $q$-space signal existing along the surface of the hypersphere. According to Fourier analysis, any square-integrable function defined on a sphere can be expanded in terms of the spherical harmonics. Thus, stereographic projection allows the $3 \mathrm{D} q$-space signal to be expanded in terms of the HSH:

$$
E_{p_{o}}(\beta, \theta, \phi) \approx \sum_{n=0}^{N} \sum_{l=0}^{n} \sum_{m=-l}^{l} C_{n l m} Z_{n l}^{m}(\beta, \theta, \phi),
$$

where $E_{p_{o}}$ denotes the $q$-space signal existing on hypersphere of radius $p_{o}$. The realness of the diffusion signal requires use of the real $\mathrm{HSH}$, and so we employ a modified real basis proposed in [6] for $Y_{l}^{m}$.

An important axiom to state is that the $q$-space signal, itself, remains invariant after the mapping - that is, for a given $q$-space point $\left(q_{x}, q_{y}, q_{z}\right)$ and its corresponding projection on the hypersphere $\left(s_{1}, s_{2}, s_{3}, s_{4}\right), E\left(q_{x}, q_{y}, q_{z}\right)=$ $E_{p_{o}}\left(s_{1}, s_{2}, s_{3}, s_{4}\right)$. In $q$-space, the diffusion signal is even i.e. $E\left(q_{x}, q_{y}, q_{z}\right)=$ $E\left(-q_{x},-q_{y},-q_{z}\right)$. Evenness in $q$-space doesn't necessarily translate into evenness on the hypersphere. According to Eq. (3), $\left(-q_{x},-q_{y},-q_{z}\right)$ projects to $\left(-s_{1},-s_{2},-s_{3}, s_{4}\right)$, and so evenness in $q$-space is tantamount to $E_{p_{o}}\left(s_{1}, s_{2}, s_{3}, s_{4}\right)$ $=E_{p_{o}}\left(-s_{1},-s_{2},-s_{3}, s_{4}\right)$ on the hypersphere, indicating that the signal is not even on the hypersphere. In other words, stereographic projection destroys 
evenness. For this reason, we are free to use both the even and odd HSH. Thus, for a given truncation order $N$, the total number of expansion coefficients is $W=(N+1)(N+2)(2 N+3) / 6$.

\subsection{Relationship between EAP and q-space Signal on Hypersphere}

Lets project 3D EAP-space onto a 4D hypersphere of radius $p_{o}$, whose coordinates are defined by the $4 \mathrm{D}$ vector $\mathbf{v}=\mathbf{v}\left(p_{o}, \beta^{\prime}, \theta^{\prime}, \phi^{\prime}\right)$. The Fourier relationship given in Eq. (1) between the signal and EAP does not hold true on the hypersphere. The question, then, is what integral transform maps from the signalhypersphere to the EAP-hypersphere. We show 1 , for the first time, that this integral transform is

$$
P_{p_{o}}(\mathbf{v})=p_{o}^{3} \int E_{p_{o}}(\mathbf{s}) \frac{e^{-2 \pi i\left[\mathbf{s} \cdot \mathbf{v}-s_{4} v_{4}\right] /(1-\cos \beta)\left(1-\cos \beta^{\prime}\right)}}{(1-\cos \beta)^{3}} d \Omega,
$$

where $P_{p_{o}}$ denotes the EAP existing on hyphersphere of radius $p_{o}$. Eq. (6) is not one of the more familiar integral transforms encountered in mathematics literature.

\subsection{HSH Metrics}

A well-known $q$-space metric is $P o \equiv P(\mathbf{k}=0)$ [1, 9, which is a measure of diffusion restrictivity. $\mathbf{k}=0$ corresponds to the south pole of the EAP-hypersphere i.e. $\beta^{\prime}=\pi$. Hence using Eq. (6) and the HSH basis, we can derive a hyperspherical Po:

$$
P o=P_{p_{o}}\left(\beta^{\prime}=\pi\right)=\int_{\Omega \in S^{3}} \frac{E_{p_{o}}(\mathbf{s})}{(1-\cos \beta)^{3}} d \Omega=\int_{\Omega \in S^{3}}\left(\frac{q^{2}+p_{o}^{2}}{2 p_{o}}\right)^{3} E_{p_{o}}(\mathbf{s}) d \Omega
$$

The integral in (7) is difficult to evaluate analytically, which is due to the nonvolume-preserving nature of stereographic projection. To overcome this, we compute an uncorrected $P o$ by assuming $q$-space is uniformly projected onto the hypersphere:

$P o_{u n c}=\int_{\Omega \in S^{3}} E_{p_{o}}(\Omega) d V=p_{o}^{3} \sum_{n, l, m} C_{n l m} \int_{\Omega \in S^{3}} Z_{n l}^{m}(\Omega) d \Omega=\pi \sqrt{2} p_{o}^{3} C_{000}$

where we use the fact that $Z_{00}^{0}=\frac{1}{\pi \sqrt{2}}$. Pounc will, naturally, suffer from volume distortion, which is corrected for by a signal weighting operation discussed in the next section.

\footnotetext{
${ }^{1}$ For derivation, see http://brainimaging.waisman.wisc.edu/ ameer/HSH_Suppl.pdf
} 
Examples of $q$-space distance metrics include the $q$-space inverse variance (QIV) [5] and the mean squared displacement (MSD) [1, which are measures of diffusivity and only pertinent to planes. A useful distance metric defined on the hypersphere is the chordal distance [7, denoted $\chi$. Consider the two $q$-space points $Q=\left(q_{x}, q_{y}, q_{z}\right)$ and $\widehat{Q}=\left(\widehat{q}_{x}, \widehat{q}_{y}, \widehat{q}_{z}\right)$ and their corresponding projections on the hypersphere $S=\left(s_{1}, s_{2}, s_{3}, s_{4}\right)$ and $\widehat{S}=\left(\widehat{s}_{1}, \widehat{s}_{2}, \widehat{s}_{3}, \widehat{s}_{4}\right)$, respectively. Then the Euclidean (4D) distance between the projections $S$ and $\widehat{S}$ on the hypersphere is given by the chordal distance, and it can be shown that

$$
\chi=\chi(Q, \widehat{Q})=\frac{2 p_{o}^{2} \sqrt{\left(q_{x}-\widehat{q}_{x}\right)^{2}+\left(q_{y}-\widehat{q}_{y}\right)^{2}+\left(q_{z}-\widehat{q}_{z}\right)^{2}}}{\sqrt{q^{2}+p_{o}^{2}} \sqrt{\widehat{q}^{2}+p_{o}^{2}}},
$$

where $\widehat{q}=\sqrt{\widehat{q}_{x}^{2}+\widehat{q}_{y}^{2}+\widehat{q}_{z}^{2}}$. If $\widehat{Q}=\mathbf{0}$, then the distance between the projection $S$ and the south pole is $\chi_{s p} \equiv \chi(Q, 0)=\frac{2 p_{o} q}{\sqrt{q^{2}+p_{o}^{2}}}$. Likewise, if $\widehat{q} \rightarrow \infty$, then the distance between the projection $S$ and the north pole is $\chi_{n p} \equiv \chi(Q, \infty)=$ $\frac{2 p_{o}^{2}}{\sqrt{q^{2}+p_{o}^{2}}}$. Geometrically, $\chi_{n p}$ and $\chi_{s p}$ are the chords on the hypersphere that form the legs of a right triangle (by Thales' theorem), with the diameter of the hypersphere the hypotenuse. The ratio of these 2 chordal lengths illuminates the relationship between the hypersphere radius $p_{o}$ and $q$-space: $p_{o}=q \frac{\chi_{n p}(q)}{\chi_{s p}(q)}(=$ constant). It can be shown that $\cos \beta=\frac{\chi_{s p}^{2}-\chi_{n p}^{2}}{4 p_{o}^{2}}$, which we define as the chordal squared difference (CSD). We then define the mean chordal squared difference (MCSD) as the CSD averaged over the surface of the signal-hypersphere i.e. $\langle\cos \beta\rangle$ :

$$
M C S D \equiv\langle\cos \beta\rangle=p_{o}^{3} \int_{\Omega \in S^{3}} \cos \beta E_{p_{o}}(\Omega) d \Omega=\frac{\pi}{\sqrt{2}} p_{o}^{3} C_{100},
$$

where we use the fact that $Z_{10}^{0}=\sqrt{2} \cos \beta / \pi$. MCSD is an inherently hyperspherical metric, whereas $P o$ is a native $q$-space metric.

\subsection{Numerical Implementation and Estimation of ODF}

Consider $M$ diffusion signal measurements (including $b=0$ ) spread across $k$ shells in $q$-space. Denote $\mathbf{G}$ as the $M \times 1$ vector representing the $M$ measurements, $\mathbf{C}$ the $W \times 1$ vector of unknown expansion coefficients $C_{n l m}$, and $\mathbf{A}$ the $M \times W$ matrix constructed with the HSH basis. Thus, we have a simple linear model of the form $\mathbf{G}=\mathbf{A C}$. This system of over-determined equations is solved via linear least squares (LLS) with Laplace-Beltrami regularization (LBR), yielding $\widehat{\mathbf{C}}=\left(\mathbf{A}^{T} \mathbf{A}+\lambda_{l} \mathbf{L}_{r e g}\right)^{-1} \mathbf{A}^{T} \mathbf{G}$, where $\mathbf{L}_{r e g}$ is the LBR diagonal matrix with entries $l^{2}(l+2)^{2}$ along the diagonal. The regularization serves to reinforce the positivity constraint of the signal.

Using Eq. (4), we correct for the volume distortion induced in Pounc by weighing each signal shell in $q$-space by $\left(\frac{q_{i}^{2}+p_{o}^{2}}{2 p_{o}}\right)^{3}$, where $q_{i}$ is the radius of the $i^{t h}$ 
shell, before signal fitting. The resulting "weighted" coefficients are then solely used for computing $P o$ via Eq. (8). The $q$-shell radii are listed in the next section. The volume weighting of each $q$-shell, in this case, can be viewed as a sampling density correction of the projected $q$-space points on the hypersphere.

Given the intricacy of Eq. (6), it is difficult to estimate the EAP analytically using the HSH framework. However, the zeroth-order diffusion ODF [8] can be numerically estimated. Lets construct a $11 \times 11 \times 11\left(-q_{\max }: \Delta q: q_{\max }\right)$ Cartesian lattice, which we map onto the $4 \mathrm{D}$ hypersphere via Eq. (3). Once we have computed the HSH expansion coefficients via LLS from the acquired data, Eq. (5) can then be used the estimate the signal at any location on the hypersphere, including the projected lattice points. Taking the fast Fourier transform (FFT) of the HSH-estimated signal for the lattice gives the EAP. The radial projection of the EAP then yields the ODF. Since the zeroth-order ODF is not inherently normalized, we min-max normalize it 8 .

\section{Experiments}

The synthetic and in vivo datasets use a hybrid, non-Cartesian $q$-space sampling scheme (HYDI) 9], consisting of 7 baseline images acquired at $b=0$ and 125 diffusion measurements spread across 5 shells in $q$-space. The number of encoding directions and $b$-value (in s/ $\mathrm{mm}^{2}$ ) for each shell are (6,300), (21,1200), (24,2700), $(24,4800)$, and $(50,7500)$; and $q_{\min }=15.79 \mathrm{~mm}^{-1}, q_{\max }=78.95 \mathrm{~mm}^{-1}$, and $\Delta q=15.79 \mathrm{~mm}^{-1}$.

Synthetic Data. Synthetic experiments were done the same way as in [5], with data generated via the bi-exponential mixture model. We look at two equally weighed fibers crossing at $45^{\circ}$, and set eigenvalues of each diffusion tensor to be $[1.6,0.4,0.4] \mathrm{e}-3$, which gives $\mathrm{FA}=0.7071$. Monte Carlo noise simulations were performed to investigate the effect of SNR on the signal reconstruction. Five SNR levels ([1 $\left.\left.\begin{array}{lllll}10 & 20 & 30 & 40 & 80\end{array}\right]\right)$ for the $b=0$ image were simulated, 1000 times each, in a similar manner as in [5], and the quality of the HSH signal fit is assessed by computing the normalized mean squared error (NMSE), given by $\frac{\|S-\widehat{S}\|^{2}}{\|S\|^{2}}$. The HSH parameters are $N=4, p_{o}=42$, and $\lambda_{l}=10^{-6}$ and those of $\mathrm{BFOR}$ are taken from [5]. In the signal fitting, HSH expends $W=55$ parameters while BFOR uses 90.

Fig. 1 displays the HSH signal fit for each shell and the corresponding ground truth in absence of noise, and shows that the HSH basis fits the diffusion signal nearly perfectly across all $b$-values. Fig. 2 displays the results of the noise simulations, with the NMSE plotted against SNR for each shell, and shows that the HSH and BFOR bases have a nearly identical robustness to noise. Fig. 3 shows the ground truth, HSH-estimated, and BFOR ODF profiles in absence of noise, indicating that the HSH basis succesfully captures the geometry and orientation of the ODF profile. However, as with the Laplacian modeling framework in diffusion propagator imaging (DPI) [2], the HSH basis smoothens the ODF peaks. The BFOR-estimated ODF is not as accurate as that of HSH. 


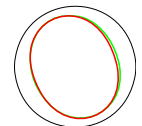

(a) $b=300$

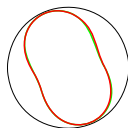

(b) $b=1200$

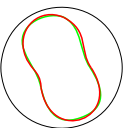

(c) $b=2700$

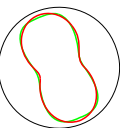

(d) $b=4800$

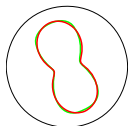

(e) $b=7500$

Fig. 1. The ground truth diffusion signal (green) and reconstructed signal (red) using HSH basis in absence of noise. Two equally weighted WM fibers were simulated crossing at $45^{\circ}$. Measurements from all 5 shells were used.

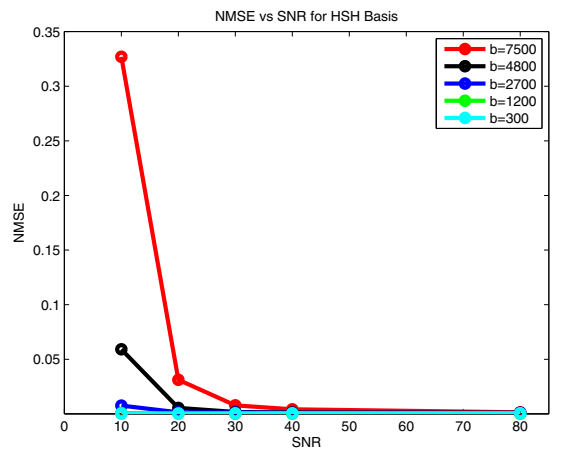

(a) NMSE for HSH basis

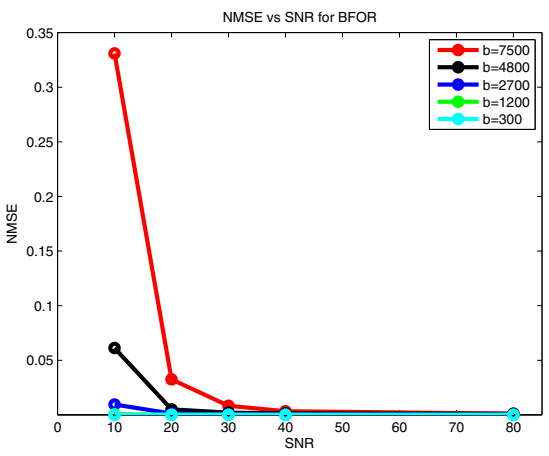

(b) NMSE for BFOR

Fig. 2. The normalized mean squared error (NMSE) of the HSH signal fit, left, and BFOR signal fit, right, for each $b$-value plotted against SNR. 1000 noise trials were simulated for each SNR level for two equally weighted fibers crossing at $45^{\circ}$.

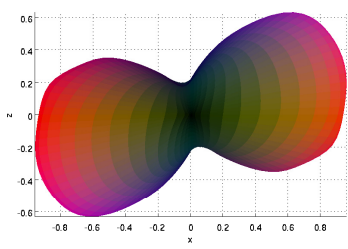

(a) HSH ODF

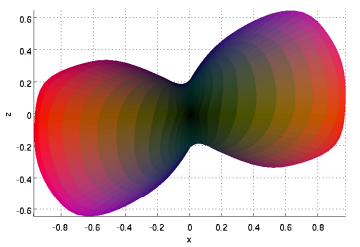

(b) Ground Truth ODF

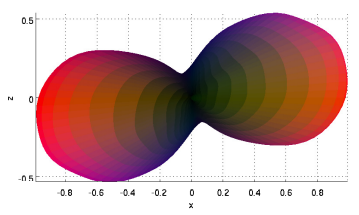

(c) BFOR ODF

Fig. 3. The HSH-estimated ODF, ground truth ODF, and BFOR ODF in absence of noise for two equally weighted fibers crossing at $45^{\circ}$. The ODF is normalized to [0 1]. 


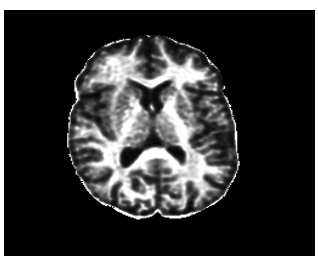

(a) HSH Po

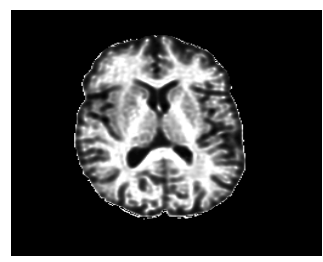

(b) BFOR Po

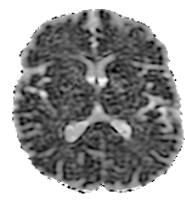

(c) MCSD

Fig. 4. Axial slices of Po, computed via HSH and BFOR bases, and MCSD maps for a healthy, adult human

Real Data. HYDI was performed on a healthy, adult human using a $3 \mathrm{~T}$ GESIGNA whole body scanner. MR parameters were $\mathrm{TE}=102 \mathrm{~ms}$, $\mathrm{TR}=6500 \mathrm{~ms}$, $\mathrm{FOV}=24 \mathrm{~cm}$, matrix $=96 \times 96$, voxel size $=2.5 \times 2.5 \mathrm{~mm}^{2}, 43$ slices with slice thickness $=3 \mathrm{~mm}$, and scan time $=15 \mathrm{~min}$. Diffusion parameters were $\delta=37.86 \mathrm{~ms}$ and $\Delta=43.1 \mathrm{~ms}$.

Axial slices of Po, computed via HSH and BFOR bases, and MCSD are shown in Fig. 4. The Po map closely resembles BFOR's, exhibiting both tissue/CSF and WM/GM constrasts. The HSH Po map, however, has sharper WM/GM contrast than BFOR's (compare the left and right putamen in both maps), which probably arises from the signal weighting operation. The MCSD map has tissue/CSF contrast but very little WM/GM contrast, and interestingly, closely resembles a mean squared displacement map. The MCSD can be viewed as a hyperspherical diffusivity measure, and specifically, an index of isotropic diffusion in neural tissue.

\section{Discussion}

We have introduced a new orthonormal basis to model the 3D $q$-space signal, and from which various metrics can be analytically derived. 4D HSH signal modeling allows for the capture of the radial and angular contents of the diffusion profile by a single basis function, and the basis' orthonormality provides robust numerical stability. The HSH basis' ability to give as good a signal reconstruction as BFOR and better ODF reconstruction, but with less fitting parameters, implies that it may be better suited to sparser sampling schemes than BFOR. Major drawbacks of the hyperspherical interpretation of $q$-space, however, are the destruction of the $q$-space signal's symmetry by stereographic projection and the difficulty in estimating the EAP via Eq. 6. Future work for HSH signal expansion includes implementing it on sparser $q$-space sampling schemes, estimating the EAP, and imposing a symmetry constraint. 


\section{References}

1. Assaf, Y., Mayk, A., Cohen, Y.: Displacement imaging of spinal cord using q-space diffusion-weighted MRI. Magn. Reson. Med. 44, 713-722 (2000)

2. Descoteaux, M., Deriche, R., LeBihan, D., Mangin, J.F., Poupon, C.: Multiple qshell diffusion propagator imaging. Med. Image Anal. 15, 603-621 (2011)

3. Domokos, G.: Four-dimensional symmetry. Physical Review 159, 1387-1403 (1967)

4. Fock, V.: Zur theorie des wasserstoffatoms. Z. Physik 98, 145-154 (1935)

5. Hosseinbor, A.P., Chung, M.K., Wu, Y.C., Alexander, A.L.: Bessel Fourier orientation reconstruction (BFOR): an analytical diffusion propagator reconstruction for hybrid diffusion imaging and computation of q-space indices. NeuroImage 64, 650$670(2013)$

6. Koay, C.G., Ozarslan, E., Basser, P.J.: A signal transformational framework for breaking the noise floor and its applications in MRI. J. Magn. Reson. 197, 108-119 (2009)

7. Saff, E.B., Snider, A.D.: Fundamentals of Complex Analysis with Applications to Engineering and Science, 3rd edn. Pearson Education, Inc. (2003)

8. Tuch, D.S.: Q-ball imaging. Magn. Reson. Med. 52, 1358-1372 (2004)

9. Wu, Y.C., Alexander, A.L.: Hybrid diffusion imaging. NeuroImage 36, 617-629 (2007) 